\title{
All optical wavelength conversion via cross phase modulation in chalcogenide glass rib waveguides
}

\author{
Vahid G. Ta'eed, Michael R. E. Lamont, David J. Moss, Benjamin J. Eggleton \\ Centre for Ultrahigh-bandwidth Devices for Optical Systems (CUDOS), School of Physics, \\ University of Sydney, NSW 2006, Australia \\ vahid@physics.usyd.edu.au \\ http://www.physics.usyd.edu.au/cudos/
}

\author{
Duk-Yong Choi, Steve Madden and Barry Luther-Davies \\ Centre for Ultrahigh-bandwidth Devices for Optical Systems (CUDOS), Laser Physics Centre, \\ The Australian National University, Canberra, ACT 0200, Australia
}

\begin{abstract}
We demonstrate all-optical wavelength conversion in a $5 \mathrm{~cm}$ $\mathrm{As}_{2} \mathrm{~S}_{3}$ chalcogenide glass rib waveguide with $5.4 \mathrm{ps}$ pulses over a wavelength range of $10 \mathrm{~nm}$ near $1550 \mathrm{~nm}$. We present frequency resolved optical gating (FROG) measurements that show good converted pulse integrity in terms of amplitude and phase in the frequency and time domains. The short interaction length ensures that dispersion induced walkoff does not hinder the conversion range of the device.
\end{abstract}

(C2006 Optical Society of America

OCIS codes: (060.4510) Optical communications; (070.4340) Nonlinear optical signal processing; (130.3120) Integrated optics devices; (190.2620) Frequency conversion

\section{References and Links}

1. O. Leclerc, B. Lavigne, E. Balmefrezol, P. Brindel, L. Pierre, D. Rouvillain, and F. Seguineau, "Optical regeneration at $40 \mathrm{~Gb} / \mathrm{s}$ and beyond," J Lightwave Technol. 21, 2779-2790 (2003).

2. Z. J. Huang, A. Gray, I. Khrushchev, and I. Bennion, "10-Gb/s transmission over $100 \mathrm{~mm}$ of standard fiber using 2R regeneration in an optical loop mirror," IEEE Photon. Technol. Lett. 16, 2526-2528 (2004).

3. J. M. Harbold, F. O. Ilday, F. W. Wise, J. S. Sanghera, V. Q. Nguyen, L. B. Shaw, and I. D. Aggarwal, "Highly nonlinear As-S-Se glasses for all-optical switching," Opt. Lett. 27, 119-121 (2002).

4. R. E. Slusher, G. Lenz, J. Hodelin, J. Sanghera, L. B. Shaw, and I. D. Aggarwal, "Large Raman gain and nonlinear phase shifts in high-purity As2Se3 chalcogenide fibers," J. Opt. Soc. Am. B 21, 1146-1155 (2004).

5. F. Ohman, S. Bischoff, B. Tromborg, and J. Mork, "Semiconductor devices for all-optical regeneration," in Proceedings of 2003 International Conference on Transparent Optical Networks, M. Marciniak, ed. (Warsaw, Poland, 2003), pp. 41-42.

6. M. Asobe, "Nonlinear optical properties of chalcogenide glass fibers and their application to all-optical switching," Opt. Fiber Technol. 3, 142-148 (1997).

7. V. G. Ta'eed, M. Shokooh-Saremi, L. B. Fu, D. J. Moss, M. Rochette, I. C. M. Littler, B. J. Eggleton, Y. L. Ruan, and B. Luther-Davies, "Integrated all-optical pulse regenerator in chalcogenide waveguides," Opt. Lett. 30, 2900-2902 (2005).

8. V. G. Ta'eed, L. Fu, M. Pelusi, M. Rochette, I. C. M. Littler, D. J. Moss, and B. J. Eggleton, "Error Free Wavelength Conversion in Highly Nonlinear Singlemode As-Se Chalcogenide Fiber," Opt. Express, Accepted (2006).

9. J. H. Lee, T. Tanemura, K. Kikuchi, T. Nagashima, T. Hasegawa, S. Ohara, and N. Sugimoto, "Use of 1-m $\mathrm{Bi} 2 \mathrm{O} 3$ nonlinear fiber for $160-\mathrm{Gbit} / \mathrm{s}$ optical time-division demultiplexing based on polarization rotation and a wavelength shift induced by cross-phase modulation," Opt. Lett. 30, 1267-1269 (2005).

10. J. H. Lee, T. Nagashima, T. Hasegawa, S. Ohara, N. Sugimoto, and K. Kikuchi, "Wavelength conversion of $160 \mathrm{Gbit} / \mathrm{s}$ OTDM signal using bismuth oxide-based ultra-high nonlinearity fibre," Electron. Lett. 41, 918-919 (2005).

11. Y. L. Ruan, W. T. Li, R. Jarvis, N. Madsen, A. Rode, and B. Luther-Davies, "Fabrication and characterization of low loss rib chalcogenide waveguides made by dry etching," Opt. Express 12, 51405145 (2004).

12. M. Shokooh-Saremi, V. G. Ta'eed, N. J. Baker, I. C. M. Littler, D. J. Moss, B. J. Eggleton, Y. L. Ruan, and B. Luther-Davies, "High-performance Bragg gratings in chalcogenide rib waveguides written with a modified Sagnac interferometer," J. Opt. Soc. Am B 23, 1323-1331 (2006).

13. D. P. Wei, T. V. Galstian, I. V. Smolnikov, V. G. Plotnichenko, and A. Zohrabyan, "Spectral broadening of femtosecond pulses in a single-mode As-S glass fiber," Opt. Express 13, 2439-2443 (2005). 
14. O. V. Sinkin, R. Holzlohner, J. Zweck, and C. R. Menyuk, "Optimization of the split-step Fourier method in modeling optical-fiber communications systems," J Lightwave Technol. 21, 61-68 (2003).

15. B. E. Olsson, P. Ohlen, L. Rau, and D. J. Blumenthal, "A simple and robust 40-Gb/s wavelength converter using fiber cross-phase modulation and optical filtering," IEEE Photon. Technol. Lett. 12, 846-848 (2000).

16. L. B. Fu, M. Rochette, V. G. Ta'eed, D. J. Moss, and B. J. Eggleton, "Investigation of self-phase modulation based optical regeneration in single mode As2Se3 chalcogenide glass fiber," Opt. Express 13, 7637-7644 (2005).

17. V. R. Almeida, R. R. Panepucci, and M. Lipson, "Nanotaper for compact mode conversion," Opt. Lett. 28, 1302-1304 (2003).

18. A. C. Turner, C. Manolatou, B. S. Schmidt, M. Lipson, M. A. Foster, J. E. Sharping, and A. L. Gaeta, "Tailored anomalous group-velocity dispersion in silicon channel waveguides," Opt. Express 14, 43574362 (2006).

19. N. J. Baker, H. W. Lee, I. C. Littler, C. M. d. Sterke, B. J. Eggleton, D.-Y. Choi, S. Madden, and B. Luther-Davies, "Sampled Bragg gratings in chalcogenide (As2S3) rib-waveguides," Opt. Express 14, 9451-9459 (2006).

\section{Introduction}

All-optical signal processing, including signal regeneration, is critical for ultra-high bit rate communications systems $[1,2]$ since current electronic processing speeds are approaching fundamental limits near $40 \mathrm{~Gb} / \mathrm{s}$. However, challenges still remain to improve device performance for practical applications. These include reducing footprint size, required optical power levels and device performance in terms of nonlinear transfer characteristics.

Chalcogenide glass offers many advantages as a platform both for fiber and integrated all optical devices, such as its large Kerr nonlinearity (up to $1000 \times$ silica glass [3]) very low two-photon absorption (TPA), photosensitivity to visible light, and an intrinsic response time less than $100 \mathrm{fs}$ [4]. This is much faster than devices based on real (as opposed to virtual) carrier dynamics such as semiconductor optical amplifiers [5]. These advantages have motivated a number of groups to investigate chalcogenide glass fiber as a nonlinear medium for self-phase modulation and Raman gain [4] and as a basis for all-optical devices, including all-optical switching [6]. In addition, there is a strong interest in developing integrated alloptical devices that would potentially offer many of the advantages that silicon integrated circuits have offered the electronics world for the past 40 years.

Recently we demonstrated an all-optical regenerator based on a chalcogenide glass rib waveguide integrated with Bragg grating filters [7]. In addition we have demonstrated wavelength conversion via cross-phase modulation in AsSe fiber [8]. In this paper, we present the first demonstration of all-optical wavelength conversion in chalcogenide planar waveguides $\left(\mathrm{As}_{2} \mathrm{~S}_{3}\right)$ via cross-phase modulation (XPM). We achieve wavelength conversion over a $10 \mathrm{~nm}$ wavelength range in a $5 \mathrm{~cm}$ long waveguide with 5.4 ps optical pulses near $1550 \mathrm{~nm}$ in the telecommunications C-band wavelength range $(1530-1570 \mathrm{~nm})$. We also present frequency resolved optical gating (FROG) measurements showing that both the temporal and phase integrity of the pulse is maintained to a high degree. These results represent a significant step in the quest for complex all-optical photonic integrated circuits for ultra-high bandwidth telecommunications.

Figure 1 shows the principle of operation of the device. A pulsed pump source, potentially containing digital data, is directed though the nonlinear medium along with a $\mathrm{CW}$ probe beam near the desired output wavelength. The pump beam induces a transient chirp on the probe beam via XPM through the Kerr nonlinearity. This broadens the probe spectra generating
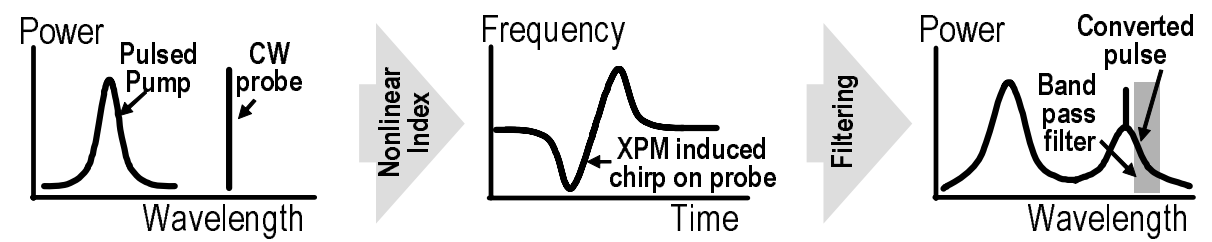

Fig. 1. Principle of wavelength conversion by cross phase modulation

\#73892 - \$15.00 USD

(C) 2006 OSA
Received 18 September 2006; revised 19 October 2006; accepted 24 October 2006 13 November 2006 / Vol. 14, No. 23 / OPTICS EXPRESS 11243 


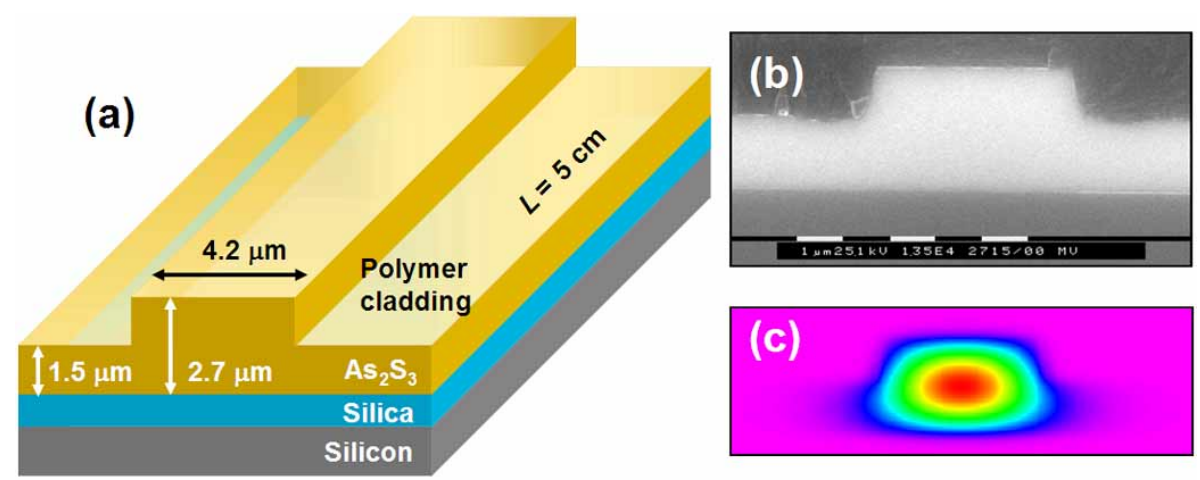

Fig. 2. Chalcogenide glass $\left(\mathrm{As}_{2} \mathrm{~S}_{3}\right)$ waveguide. (a) Schematic, (b) scanning electron micrograph (SEM) and (c) simulated mode profile.

sidebands, and when a single sideband is selected using an optical filter, the output signal at the converted wavelength is modulated in time similarly to the pump pulse. This principle has also been recently used for regeneration of noisy signals and wavelength conversion in $\mathrm{Bi}_{2} \mathrm{O}_{3}$ fiber $[9,10]$.

\section{Experiment}

Figures 2(a-b) show a schematic and a scanning electron micrograph (SEM) of the rib waveguide cross section. $2.5 \mu \mathrm{m}$ thick $\mathrm{As}_{2} \mathrm{~S}_{3}$ film was deposited via pulsed laser deposition [11] onto a silica-on-silicon substrate comprised of a $2.5 \mu \mathrm{m}$ thick silica layer. Rib waveguides were fabricated by photolithography and inductively coupled plasma reactive ion etching with $\mathrm{CF}_{4}-\mathrm{O}_{2}$ gas. The wafers were dehydration baked at $180^{\circ} \mathrm{C}$ for 2 minutes on a hot plate before photoresist coating. The waveguide width was $4.7 \mu \mathrm{m}$ and etch depth of $1.2 \mu \mathrm{m}$, as shown in Fig. 2(a). After resist removal with standard wet stripping, the waveguides were clad with a UV cured inorganic polymer glass (RPO Pty Ltd, IPG ${ }^{\mathrm{TM}}$ ) which has a refractive index of 1.53 at $1550 \mathrm{~nm}$. End facets were then prepared on the waveguides by hand cleaving the silicon substrate with a diamond scribe, resulting in $5 \mathrm{~cm}$ long waveguides.

As the $\mathrm{As}_{2} \mathrm{~S}_{3}$ rib waveguide fabrication is still at an early stage, the current batch showed varying levels of high polarization dependent loss (PDL), although low PDL has been achieved previously [11]. Earlier work [12] utilizing Bragg gratings to interrogate the waveguide modal properties showed varying birefringence and inferred that fabrication processes may be involved. For this experiment we utilized the low propagation loss TE waveguide mode. The mode profile, Fig. 2(c), was simulated by beam propagation method and had an effective core area of $7.2 \mu \mathrm{m}^{2}$.

The experimental configuration for wavelength conversion is shown in Fig. 3. Pulses from a passively modelocked "Figure 8 " laser $(1549.6 \mathrm{~nm}$ wavelength, pulse width $5.4 \mathrm{ps,}$

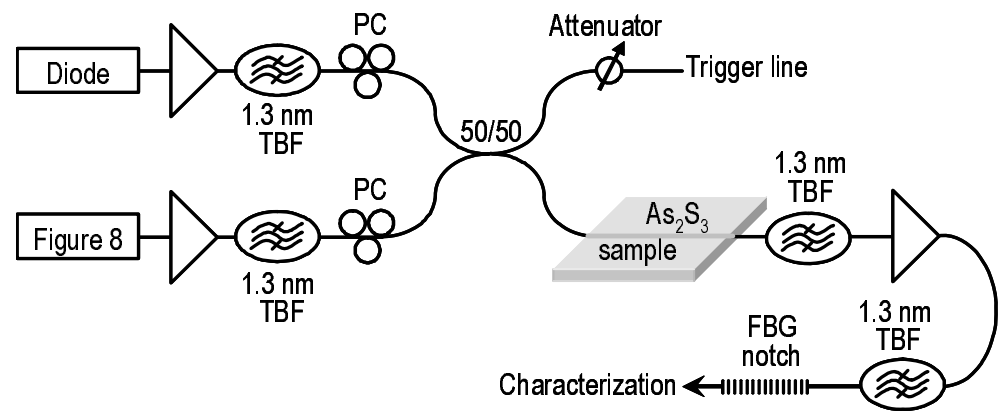

Fig. 3. Experimental setup for demonstrating cross phase modulation based wavelength conversion.

$\# 73892$ - \$15.00 USD

(C) 2006 OSA
Received 18 September 2006; revised 19 October 2006; accepted 24 October 2006 13 November 2006 / Vol. 14, No. 23 / OPTICS EXPRESS 11244 
repetition rate $9 \mathrm{MHz}$ ) were amplified and combined with a CW probe from a wavelength tunable amplified laser diode using a 50-50 coupler. Tunable bandpass filters (TBF $-3 \mathrm{~dB}$ width $1.3 \mathrm{~nm}$ ) were used to remove out-of-band amplified spontaneous emission (ASE) from both the pulsed pump and CW probe. The combined pump and probe were coupled into the waveguide via standard SMF fiber with a short $(5 \mathrm{~mm}$ ) high numerical aperture fiber (HNAF) segment $(4 \mu \mathrm{m}$ MFD) spliced on the end to improve coupling losses. Silica index matching oil reduced Fresnel reflections at the silica/ $\mathrm{As}_{2} \mathrm{~S}_{3}$ interface. Total insertion loss of the waveguide, including SMF/HNAF splices was 5 to $6 \mathrm{~dB}$, with propagation losses typically of $0.2 \mathrm{~dB} / \mathrm{cm}$. In-line polarization controllers ensured that the polarization state of the pulse pump and $\mathrm{CW}$ probe were aligned.

The output of the $\mathrm{As}_{2} \mathrm{~S}_{3}$ waveguide was then sent through a $1.3 \mathrm{~nm}(3 \mathrm{~dB})$ tunable bandpass filter offset from the $\mathrm{CW}$ probe by $1.0 \mathrm{~nm}$ to remove the pump and probe, and select a single XPM sideband on the long wavelength side. After amplification, any residual CW carrier was removed with a $200 \mathrm{pm}$ wide fiber Bragg grating (FBG) notch filter, and the signal was directed to measurement apparatus. For characterization, the signal was amplified and filtered with the TBF to remove out-of-band ASE, and then directed to either an optical sampling oscilloscope (10 GHz bandwidth), a Frequency Resolved Optical Gating (FROG) apparatus for both temporal and phase measurements, or an optical spectrum analyzer.

Peak pump powers within the sample were kept to $\sim 36 \mathrm{~W}$ or below. This corresponded to a maximum intensity of less than $500 \mathrm{MW} / \mathrm{cm}^{2}$, which is below the photo-darkening damage threshold reported for this material [13]. The typical in fiber average powers utilized for the pump and probe was $3.5 \mathrm{~mW}$ and $120 \mathrm{~mW}$ respectively. The large average probe power was required due to the low duty-cycle of the pump used in this experiment.

\section{Results}

Figure 4(a) shows the spectra directly after the waveguide of the unfiltered output signal containing both the pump ( $2 \mathrm{~nm}$ wide signal at $1549.7 \mathrm{~nm})$ and $\mathrm{CW}$ probes set to three different wavelengths $(1555.5 \mathrm{~nm}, 1559.6 \mathrm{~nm}$ and $1563.7 \mathrm{~nm})$. The sidebands on each probe are clearly visible. Note that the strength of the sidebands are proportionately reduced because of the extremely low duty cycle $(5.4$ ps pulses at $9 \mathrm{MHz})$ used in these experiments. For a full $33 \% \mathrm{RZ}$ signal the relative strength of the sidebands would be increased by $35 \mathrm{~dB}$. Also shown in Fig. 4(b) are simulations of the output spectra obtained using a symmetrized splitstep Fourier method with an adaptive step-size [14] with an $n_{2}$ of $100 \times$ silica, in agreement with earlier work $[7,11]$. We calculate that the input signal pulses generate a nonlinear phase shift of 3.0 radians.

It is well known that group velocity mismatch (i.e. walk-off) between the pump and probe results in a reduced interaction length. For $T_{0}$, the exponential half width of the pulse, the walk-off length is given by $L_{\mathrm{W}}=T_{0} /(D \Delta \lambda)$, where $\Delta \lambda$ is the pump-probe wavelength offset and $D$ is the dispersion parameter which is $-400 \mathrm{ps} / \mathrm{km} \mathrm{nm}$ at $1550 \mathrm{~nm}$ [6]. For interaction lengths greater than the walk-off length, asymmetry can occur in the XPM generated sideband spectra. Experimentally, the apparent observed asymmetry in the output sidebands shown in Fig. 4(a) is caused by the ASE pre-filter on the CW probe, offset to maximize ASE extinction on the long wavelength side. Calculations show that walk-off is only significant for wavelength offsets as large as $100 \mathrm{~nm}$. This is primarily because the $\mathrm{As}_{2} \mathrm{~S}_{3}$ rib waveguide length $(5 \mathrm{~cm})$ is significantly shorter than other demonstrations (e.g. $5 \mathrm{~km}$ of silica [15], bismuth oxide [10] or As-Se [8] fiber).

Figure 4(c) shows the filtered spectra of the pulses converted to three different wavelengths $(1556.4 \mathrm{~nm}, 1560.5 \mathrm{~nm}$ and $1564.7 \mathrm{~nm})$. Interpolating the background ASE level shows an approximately $10 \mathrm{~dB}$ of optical signal to noise ratio for the three converted pulses. However, this is deceiving due to the low duty cycle of the pump laser. In terms of actual converted pulse peak power relative to background ASE, this is in fact $\sim 53 \mathrm{~dB}$.

$\# 73892$ - \$15.00 USD

(C) 2006 OSA
Received 18 September 2006; revised 19 October 2006; accepted 24 October 2006 13 November 2006 / Vol. 14, No. 23 / OPTICS EXPRESS 11245 

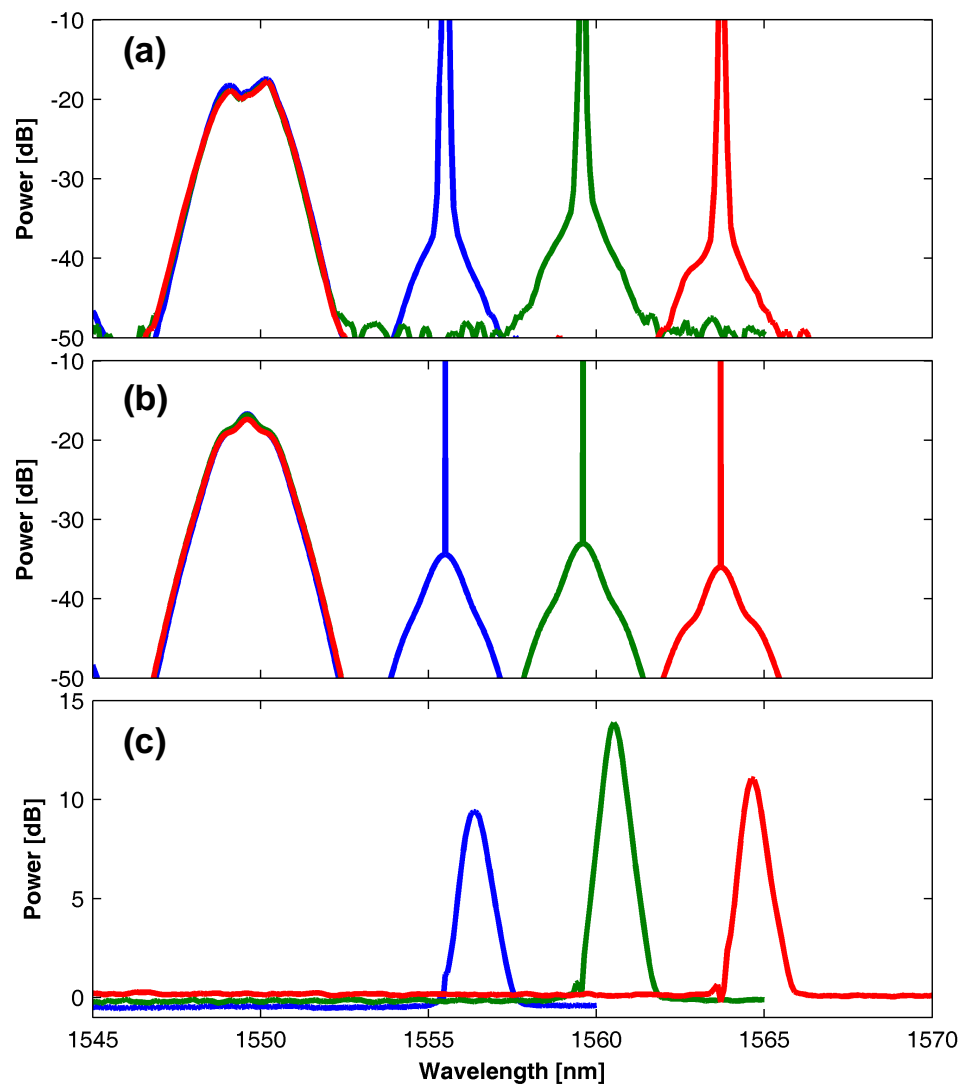

Fig. 4. (a) Experimental and (b) simulated unfiltered output spectra of signal from waveguide showing both pulsed pump and three different CW probes with XPM sidebands imprinted on them. (c) Experimental filtered output spectra of device leaving behind only a single sideband.
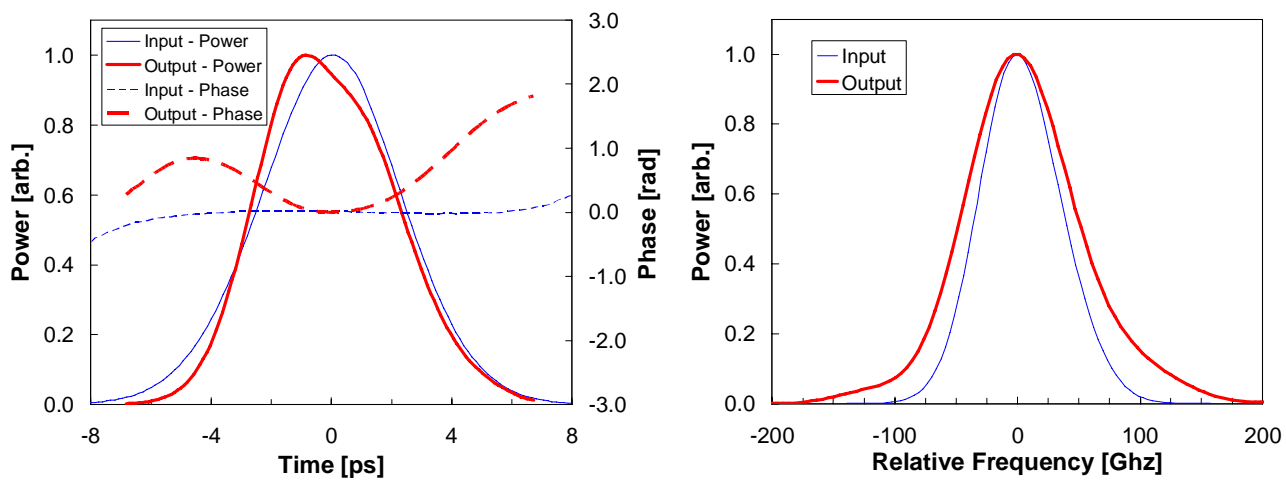

Fig. 5. Frequency resolved optical gating (FROG) results for both the temporal response (right) and spectrum (left) of the input and output pulses.

In order to characterize both the temporal and spectral amplitude and phase performance of this device we measured the output pulses via frequency resolved optical gating (FROG),

\#73892 - \$15.00 USD

(C) 2006 OSA 
and the results are shown in Fig. 5. The output pulse experiences very little temporal amplitude distortion over the input pulse (Fig. 5(a)). The temporal phase however experiences a degree of quadratic distortion, leading to a small amount of chirp in the output pulse. The effect of this on the output spectra, however, is modest. Figure 5(b) shows the input versus output spectra as measured with the FROG system, showing that the induced chirp results in spectral broadening on the order of $24 \%$ - still fairly close to transform limited.

\section{Discussion}

We were able to obtain results in our $\mathrm{As}_{2} \mathrm{~S}_{3}$ rib waveguide device because of the small mode field area of $7.2 \mu \mathrm{m}^{2}$ combined with a high Kerr nonlinear index $n_{2}=3.05 \times 10^{-14} \mathrm{~cm}^{2} / \mathrm{W}$ $(\sim 100 \times$ silica and 3-4 times larger than bismuth oxide) resulting in a large nonlinear coefficient of $\gamma \sim 1700 \mathrm{~W}^{-1} \mathrm{~km}^{-1}$ [16]. In addition, as the dispersion remains relatively constant over the entire communications band, this $\mathrm{As}_{2} \mathrm{~S}_{3}$ based device can in principle be reconfigured for use in any telecommunication band, in contrast with dispersion shifted silica fiber, for example.

The strong nonlinearity exhibited by these waveguides can also result in other nonlinear effects. Spectrally, we have observed four-wave mixing. This phenomenon was approximately $10 \mathrm{~dB}$ weaker than the XPM process as the waves are not phase matched at these wavelengths. In recent work on wavelength conversion in $\mathrm{As}_{2} \mathrm{Se}_{3}$ chalcogenide glass fiber [8], we found that two photon absorption did not have a significant impact on the device performance. In this work, since $\mathrm{As}_{2} \mathrm{~S}_{3}$ has much lower TPA [6] than $\mathrm{As}_{2} \mathrm{Se}_{3}$ so we did not expect, nor did we observe, any effects of nonlinear absorption.

A key goal is demonstration at ultrahigh bit rates, for example $160 \mathrm{~Gb} / \mathrm{s}$. For a $25 \mathrm{~nm}$ conversion bandwidth, the $2.0 \mathrm{ps}$ pulse walk-off length is $7.1 \mathrm{~cm}$ corresponding to an effective length of $6.0 \mathrm{~cm}$. Power reduction can be achieved first by tailoring the material composition to increase the nonlinear index; $\mathrm{As}_{2} \mathrm{Se}_{3}$ has a nonlinear index 400 times that of silica [16]. Second, the effective core area can be significantly reduced; a $700 \mathrm{~nm}$ wide by $300 \mathrm{~nm}$ tall $\mathrm{As}_{2} \mathrm{~S}_{3}$ ridge waveguide has an effective core area of only $0.41 \mu \mathrm{m}^{2}$. These two methods would reduce the peak operating power to only $\sim 0.43 \mathrm{~W}$. Low-loss coupling to such a waveguide could be achieved using an inverse taper structure [17].

As a side note, it is worth mentioning that tight mode field confinement also results in significant waveguide dispersion which could be utilized to compensate for the large material dispersion as has been achieved with highly dispersive silicon [18] where the intrinsic $\mathrm{D}=-880 \mathrm{ps} / \mathrm{nm} / \mathrm{km}$. This would allow either increased operating bandwidth or utilization of nonlinear parametric processes.

Finally, current work is in progress to integrate some of the required optical filters into the waveguide in a similar fashion to what was reported for an integrated $2 \mathrm{R}$ regenerator [7]. Although filter requirements for this application are considerably more stringent, complex gratings have been achieved [19] and so we do not foresee any fundamental road blocks to achieving a fully integrated all optical wavelength converter.

\section{Conclusions}

We report the first demonstration of all-optical wavelength conversion in chalcogenide glass planar waveguides. We achieve near perfect conversion over a $10 \mathrm{~nm}$ range near $1550 \mathrm{~nm}$ in $\mathrm{As}_{2} \mathrm{~S}_{3}$ rib waveguides fabricated by pulsed laser deposition and reactive ion etching, using cross phase modulation of a wavelength tuneable $\mathrm{CW}$ probe by $5.4 \mathrm{ps}$ pump pulses. Dispersive walk-off effects are minimized in the short device, ensuring wide bandwidth operation. Calculations indicate a straightforward route towards implementing an ultrahigh bit-rate device.

\section{Acknowledgments}

This work was produced with the assistance of the Australian Research Council (ARC). CUDOS (the Centre for Ultrahigh-bandwidth Devices for Optical Systems) is an ARC Centre of Excellence.

$\# 73892$ - \$15.00 USD

(C) 2006 OSA
Received 18 September 2006; revised 19 October 2006; accepted 24 October 2006 13 November 2006 / Vol. 14, No. 23 / OPTICS EXPRESS 11247 